Data Descriptor

\title{
Development of a Data Set of Pesticide Dissipation Rates in/on Various Plant Matrices for the Pesticide Properties Database (PPDB)
}

\author{
Kathleen Lewis * (D) and John Tzilivakis \\ Agriculture \& the Environment Research Unit, University of Hertfordshire, Hatfield, AL10 9AB, UK; \\ j.tzilivakis@herts.ac.uk \\ * Correspondence: k.a.lewis@herts.ac.uk; Tel.: +44-1707-284582
}

Received: 10 August 2017; Accepted: 26 August 2017; Published: 29 August 2017

\begin{abstract}
Data relating to the rate at which pesticide active substances dissipate on or within various plant matrices are important for a range of different risk assessments; however, despite the importance of this data, dissipation rates are not included in the most common online data resources. Databases have been collated in the past, but these tend not to be maintained or regularly updated. The purpose of the exercise described herein was to collate a new database in a format compatible with the main online pesticide database resource (the Pesticide Properties Database, PPDB), to validate this database in line with the Pesticide Properties Database protocols and thus ensure that the data is maintained and updated in future. Data was collated using a systematic review approach using several scientific databases. Collated literature was subjected to a quality assessment, and then data was extracted into an MS Excel spreadsheet. The outcome of the study is a database based on data collated from 1390 published articles covering over 400 pesticides and over 200 crops across a wide variety of different matrices (leaves, fruits, seeds etc.) for pesticide residues on the crop surface, as well as residues absorbed within the plant material. This data is now fully incorporated into the PPDB.
\end{abstract}

Data Set: available as a supplementary file: "Plant dissipation data August 2017.xlxs", http:/ /www. mdpi.com/2306-5729/2/3/28/s1.

Data Set License: This data set was made available under a CC-BY license.

Keywords: pesticide dissipation; risk assessment; environmental fate

\section{Summary}

Data relating to the rate at which pesticide active substances dissipate or decay on or within various plant matrices (e.g., leaves, stems, seeds, fruits) are important for a range of different risk assessments. For example, dissipation rates can be used to determine when workers can safely re-enter fields and glasshouses following a pesticide application [1], and may also be used to estimate the potential exposure of individuals who may come in contact with, for example, sprayed sports turf or golf greens [2,3]. Dissipation rates also have application in consumer safety. For example, these values are used in calculations for predicting residue concentrations in harvested produce and for determining the time interval needed between crop spraying and harvesting or potential processing/consumption in order to minimise residue concentrations [4,5]. Dissipation rates also have value when considering the potential risk to non-target and beneficial organisms (e.g., pollinators) that may forage or otherwise come in contact with a pesticide treated plant, as well as informing on how long the chemical is likely to offer satisfactory pest control before it decays [6-8]. As a consequence, plant matrix half-lives are often an important input parameter into various risk assessment models [9-12]. 
In this context, dissipation rate is defined as the rate at which the pesticide active substance disappears from the part of the plant measured due to the combined effects of different processes including volatilisation, hydrolysis, photolysis, chemical and microbial degradation etc. Dissipation rates are often determined using field studies, and they are usually expressed as the pesticide half-life $\left(R L_{50}\right)$; the half-life being defined as the time required for the pesticide residue level to fall to half of the initial concentration directly after application. Data tends to be highly variable, depending not just on the physicochemical behaviour of the active substance, but also on the type of plant matrix, its texture (e.g., presence of waxes or surface hairs), the overall plant architecture (e.g., leaf shape, fruiting habits), and if the dissipation rate is measured on the crop surface or if it has been absorbed into its flesh $[13,14]$. For any given active substance product, formulations can vary considerably, and may contain various additives that can affect how the pesticide behaves on the plant. Whether or not these additives affect residue behaviour is largely unknown, but is part of new research being undertaken both in industry and at various research institutes. In addition, climatic factors (e.g., air temperature, humidity, sunlight intensity) and environmental conditions under which the crop/plant has been grown or stored will also influence dissipation rates $[15,16]$.

Despite the fact that the importance of this type of data for risk assessments has been evident for many years, the data is not routinely reported in any of the main reference sources [17-20], nor is it normally part of the data package reported and used to support the regulatory authorisation process undertaken by the European Commission, US EPA or many other regulatory bodies in the developed world where there are comprehensive and sophisticated risk assessment processes in place. Indeed, up until the late 2000s, data was sparse, and for many applications it was estimated from the soil half-life or via vegetation models; approaches that would undoubtedly have had implications for the study outcomes [21,22]. Although there is now a considerable number of published studies available, and the number is increasing year on year, the data is still problematic to identify; the main issue being the amount of time needed to source the data, which is scattered within published scientific literature, and subsequently understand its variability and parameter sensitivity such that the most appropriate value can be used.

There have been useful attempts to collate dissipation rate data from literature in the past and to publish the collated data set. For example, Willis and McDowell [23] created a data set of dissipation rates for 79 different pesticide substances on plant leaves in 1987, Katagi [24] reported data for 65 pesticides on a variety of matrices in 2004 and, Fantke and Juraske [15] collated a much larger data set of 346 pesticides, again on a range of plant matrices using 811 published studies in 2013. All of these data sets, particularly the latter one, have been hugely valuable to the risk assessment community, but the value of these data, and indeed other risk assessment data, is time-limited. Each year, a number of new active substances are released onto the global market, whilst others are removed by regulatory processes or for commercial reasons. In addition, new research is published continually that adds to the knowledge base and provides the opportunity to better understand the data and its variability. Therefore, if a data set is to remain valuable and actively used, it is important that it is regularly updated, maintained and managed. There is also a need for it to be made easily accessible, preferably alongside other related data. This has not happened as well as it might with the data sets cited above.

The Pesticide Properties Database_PPDB [18] —is an online resource of pesticide data relating to its chemical identification, physicochemical characteristics, metabolism, environmental fate, human and ecological toxicity and regulatory status. Whilst the PPDB is widely used for a variety of applications, its primary purpose is to support risk assessments. This database is now used extensively throughout the world, with typically 3000-5000 page loads of information being downloaded daily. It is externally peer reviewed, endorsed by the International Union of Pure and Applied Chemistry (IUPAC), and promoted by several major organisations including the Food and Agriculture Organization (FAO). It is managed and maintained on an ongoing basis according to prescribed protocols. The PPDB is free of charge and free of advertising at the point of online user access. It is also 
available, under license, as an off-line MS Access or MS Excel file, and so can be linked directly to mathematical models and decision-support systems [18].

Prior to this work being conducted and despite its extensive scope, the PPDB did not contain data relating to dissipation rates on or in plant matrices, and so the aim of this study was to create a data set to plug this gap with peer-reviewed literature, building on the work of others-particularly that of Fantke and Juraske [15] — and using these earlier data sets to help verify the newly collated data. The new data set would then be incorporated into the PPDB and so become subject to the PPDB's continuous updating and management programme. The data set presented in this manuscript provides the data collated to date. This data has now been incorporated into the PPDB, and new data will be added as and when it is identified. Thus, the data is now easily accessible to the PPDB's global user base for use in risk assessments and pesticide environmental fate studies.

\section{Data Description}

The data set as presented herein has a very simple structure. It consists of a single MS Excel file comprised of 14 parameters as shown in Table 1. The data includes three parameters to aid correct chemical identification (common name, chemical name and CAS RN). Two parameters are used to describe the crop/plant (common name and scientific name, including any other distinguishing information, such as the variety or cultivar where this is known) and a further two parameters describing the matrix type (skin, leaves, fruit etc.) and whether dissipation rates were measured on the matrix surface or as a total residue (i.e., on and in the matrix). There are also two values that may be used to help identify the conditions under which the plant/crop was grown. The first is the country in which the study was undertaken, and for most instances, this is a country where production/occurrence of the crop/plant is common. The second describes whether the study was undertaken in the open field, undercover, or under particular conditions (e.g., cold storage, flood irrigation). Dissipation rates are reported as arithmetic mean for the pesticide-plant-matrix combination as reported in the published literature. However, in some incidences, whilst sufficient data has been provided within an article to calculate half-lives, only the temporal variation in pesticide concentration within or on the matrix has been reported. In these cases, half-lives have been determined via first-order kinetics using Equations (1) and (2) [15,25].

$$
\begin{gathered}
\mathrm{C}_{\mathrm{t}}=\mathrm{C}_{0} \mathrm{e}^{-\mathrm{kt}} \\
\mathrm{RL}_{50}=\ln 2 / \mathrm{k}
\end{gathered}
$$

where $C_{t}$ represents the concentration of pesticide at time $t, C_{0}$ represents the initial concentration and $\mathrm{k}$ is the pesticide dissipation rate constant in days. The half-life $\left(R L_{50}\right)$ is then determined from the $\mathrm{k}$ value for each experiment.

Where the article reports more than one experiment on the same pesticide-plant-matrix combination, the data range across experiments is reported. This approach is in contrast to that of Fantke \& Juraske [15], who report experimental RL $_{50}$ values for each separate experiment/data point, but was adopted to enable the data to be readily incorporated into the structure of the PPDB and to better facilitate direct access of the data by models and decision support systems that tend to require just a single value for each parameter. 
Table 1. Description of the data set variables.

\begin{tabular}{|c|c|}
\hline Parameter & Description \\
\hline Pesticide common name & $\begin{array}{l}\text { The name by which the pesticide active substance is commonly known. Data in } \\
\text { this column are listed alphabetically. }\end{array}$ \\
\hline Pesticide chemical name & $\begin{array}{l}\text { Chemical name of the pesticide using the Chemical Abstract Services (CAS) } \\
\text { nomenclature. }\end{array}$ \\
\hline CAS registry number & $\begin{array}{l}\text { The Chemical Abstract Services' unique identifying number (RN) assigned to } \\
\text { the pesticide. }\end{array}$ \\
\hline Plant & Common name of the plant/crop the data relates to. \\
\hline Plant scientific name & Scientific name including cultivar or variety where known \\
\hline Matrix & The part of the plant tested. \\
\hline On/In & $\begin{array}{l}\text { Whether the residue was measured on }(\mathrm{O}-\text { as a surface residue) on in ( } \mathrm{I} \text { - as } \\
\text { total residue in and on) the sample. }\end{array}$ \\
\hline Country & The country (and in some instances region) where the study was undertaken. \\
\hline Study conditions & $\begin{array}{l}\text { Whether the study was undertaken in the open field }(\mathrm{F}) \text {, undercover }(\mathrm{U}) \text { or } \\
\text { under special conditions }(\mathrm{X}) \text {. In the latter case the data is accompanied by short } \\
\text { qualifying text. }\end{array}$ \\
\hline Min DT50 (days) & $\begin{array}{l}\text { Minimum experimental value for the plant dissipation rate expressed as the } \\
\text { half-life (RL50) in days. }\end{array}$ \\
\hline Max DT50 (days) & $\begin{array}{l}\text { Maximum experimental value for the plant dissipation rate expressed as the } \\
\text { half-life }\left(\mathrm{RL}_{50}\right) \text { in days. }\end{array}$ \\
\hline Mean DT50 (days) & $\begin{array}{l}\text { Arithmetic mean experimental value for the plant dissipation rate expressed as } \\
\text { the half-life }\left(\mathrm{RL}_{50}\right) \text { in days. }\end{array}$ \\
\hline Reference & $\begin{array}{l}\text { Full bibliographical reference for the publication from which the data } \\
\text { was extracted. }\end{array}$ \\
\hline PPDB code & Unique identifier linking the record to the PPDB (see User Notes below). \\
\hline
\end{tabular}

The data set currently contains data for 407 different active substances across 207 different plants on a wide range of different matrices including leaves, fruits, seeds, root, new shoots etc., and was established from 1390 published studies. There are currently over 2200 records for unique pesticide-plant-matrix combinations. The data set clearly demonstrates the variability of the data, supporting and further enforcing the conclusions of Fantke \& Juraske [15], and the need to carefully select appropriate data for risk assessment modelling to avoid introducing errors. An example of this variability can be illustrated with the records available for the pesticide cypermethrin. According to the PPDB cypermethrin is a pyrethroid insecticide commonly used across the world to control a broad spectrum of pests such as aphids, caterpillars and damaging beetles on a wide range of crops including cereals, vegetables, oilseeds, potatoes and top fruit [18]. Figure 1 shows dissipation data for cypermethrin on the surface of a wide range of plant matrices. The range bars show the spread across the studies identified in the literature review. Across plants, the half-life on leaves varies between 2.5 and 13.1 days, whereas that on fruit varies 1.9 and 6.1 days. 


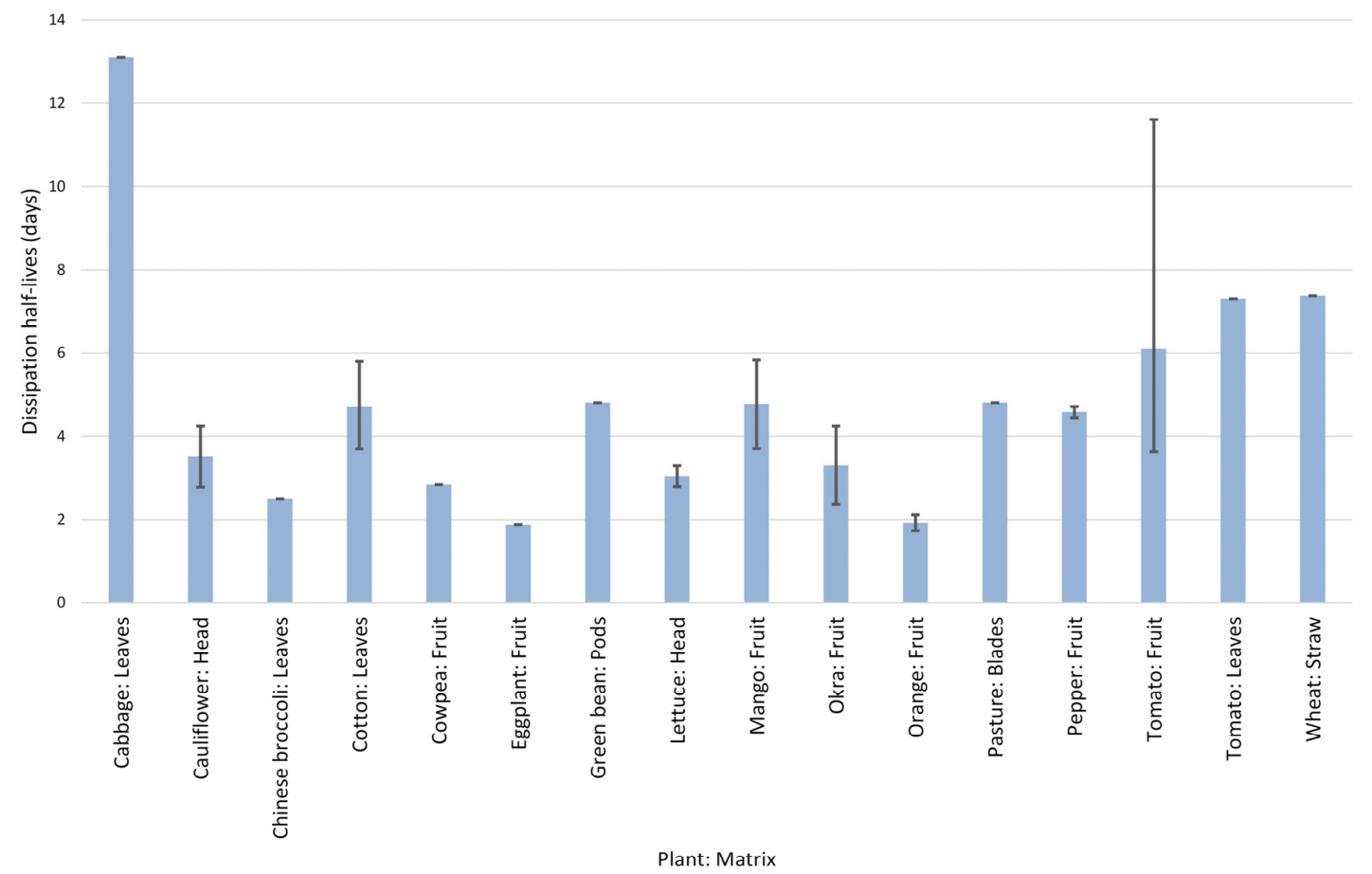

Figure 1. Variation in dissipation rate of cypermethrin on various plant matrices.

Figure 2 shows similar data but for the same pesticide in and on the crop matrix. In this instance, the half-life on leaves varies between 1.8 to 4.8 days, whereas that on fruit varies 1.3 and 11.7 days. This data is very different from that for soil degradation. According to data within the PPDB cypermethrin is quite persistent in soil and half-lives are typically 70-200 days range [18], which clearly illustrates that using soil data as a surrogate for plant matrix data is inappropriate.

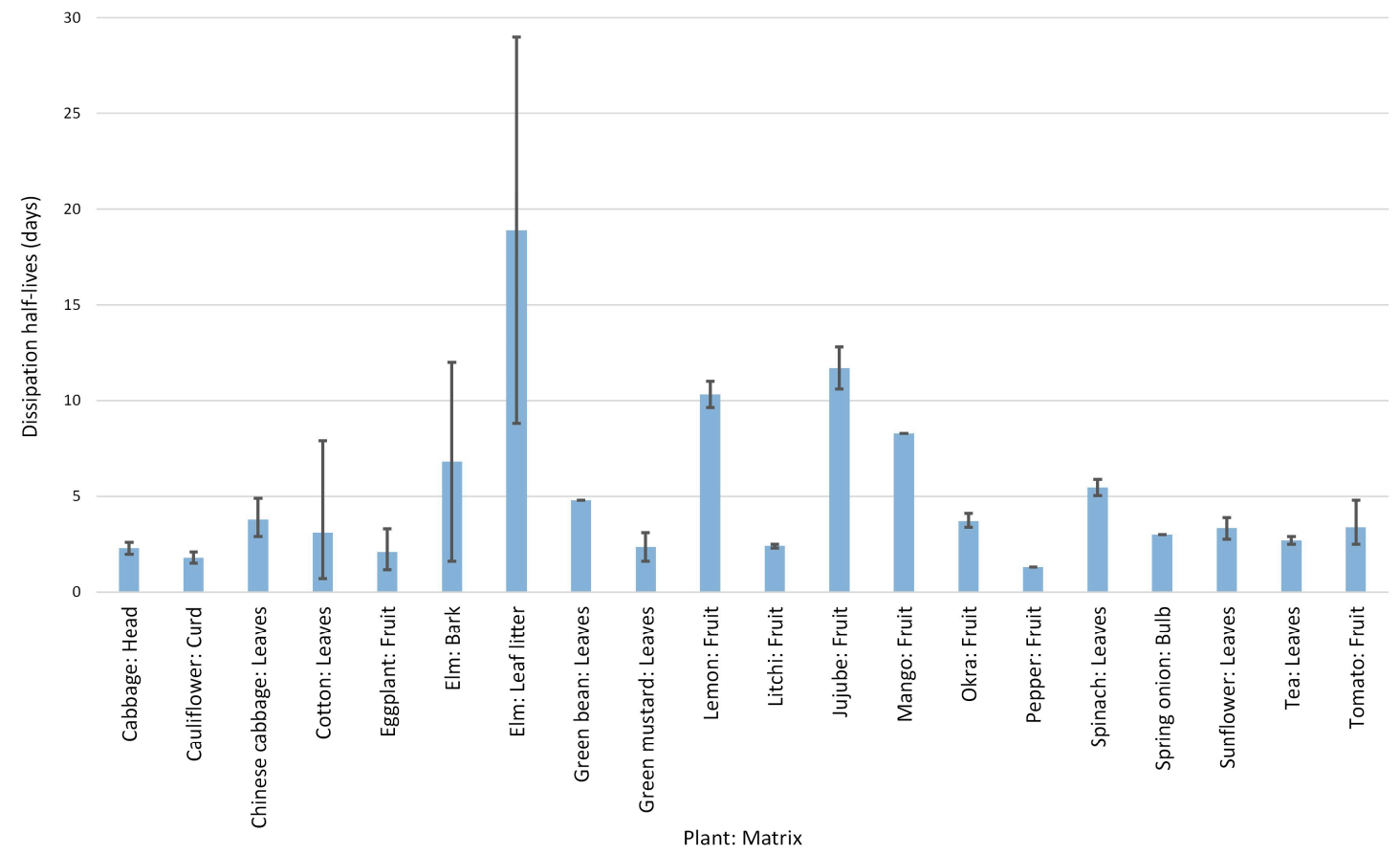

Figure 2. Variation in dissipation rate of cypermethrin on and in various plant matrices. 


\section{Methods}

Whilst the methodology adopted for this study was not strictly that of a systematic review, it did utilise a similar logical and systematic approach, in that it used a predefined plan (the review protocol) that identified the literature databases to be searched (i.e., Google Scholar, ScienceDirect, Scopus, American Chemical Society Journals Database) and included a rigorous search protocol to recognise and obtain all (insofar as this is reasonably possible) relevant peer reviewed published literature available during the period January 1980 to June 2017. The review protocol also included a number of criteria that the published study would need to comply with in order for the data to be considered of sufficient quality to be extracted. The criteria included, for example, evidence that the experimental approach was scientifically sound, that the experiments had been replicated, and that the statistical analysis was appropriate to the study. The extracted data also needed to be original and empirical, i.e., determined by experiments and not modelled or inferred in any way. The reference lists of each retrieved publication were scanned to identify additional data. If, during the course of the review, data was identified that had been published earlier than 1980, this was also included, subject to quality controls. Review articles have primarily been used to identify suitable studies and not for data extraction. However, in some instances, particularly where the article is old and obtaining a copy was problematic, data has been used that was quoted in the review article itself. Obviously, this approach is not ideal, as it is not possible to check the data nor to judge its quality. So the decision to include data from reviews was based on judgement regarding the value of the data in terms of the amount of other data available on the pesticide/plant/matrix combination.

Once suitable data had been identified, it was extracted into an MS Excel spreadsheet according to the structure given in Table 1. Simple software routines were written to assist with data validation and analysis. This included comparing the data with that in databases created by others (e.g., [15]) and subsequently identifying the cause of data disagreements, as well as checking for typographical errors and duplicates.

Future updates will be undertaken periodically using the same approach described herein and added to the PPDB according to its established protocols.

\section{User Notes}

In the data set provided as a supplementary MS Excel file, a parameter called "PPDB Code" is provided for each active substance. This is a unique code that links the data in the MS Excel file with the full data profile for that pesticide in the PPDB. The PPDB profile can be accessed using the following URL: http:/ / sitem.herts.ac.uk/aeru/ppdb/en/Reports/\#.htm after replacing the "\#" with the PPDB Code for that substance. For example, to access the full data set for cypermethrin, use the URL: http:/ / sitem.herts.ac.uk/aeru/ppdb/en/Reports/197.htm. Alternatively the PPDB homepage has an A-Z listing, as well as a search function.

Pesticide active substances are often known by many different common names and common names are often different in different languages. If the pesticide required is not found in the data set, check again using its CAS RN identifier or via an alias. A list of aliases and CAS RN identifiers for that substance can be found in the PPDB and the PPDB search function may be useful in this respect.

Within the online version of the PPDB, for each active pesticide substance and for both on and within the matrix, the mean value across plants and matrices is reported together with descriptive text describing the data variability. The full data set, as given in this article's supplementary file, is available in the MS Access and MS excel versions of the database.

Note that data relating to processed products, for example wine or "made tea", have not been included within this data set, but may be in those created by others.

Data amendments and updates will, in future, be added directly to the PPDB.

Supplementary Materials: The data set is available as a supplementary file: "Plant dissipation data August 2017.xlxs", http://www.mdpi.com/2306-5729/2/3/28/s1. 
Acknowledgments: This study was undertaken without direct funding by any organisation. It was undertaken as part of the PPDB updating process funded by PPDB licensing and consultancy fees. The authors express their gratitude to the PPDB user base for their continued support.

Author Contributions: Kathy Lewis undertook the literature review, evaluation of the studies and collated the raw data. John Tzilivakis wrote the analytical routines and subsequently used these to analyse the data, identify duplicates and errors. John Tzilivakis was also responsible for the formatting of the final data set presented with this manuscript. Both authors agree to be listed and have approved the submitted article.

Conflicts of Interest: The authors declare no conflict of interest.

\section{Abbreviations}

The following abbreviations are used in this manuscript:

CAS RN Chemical Abstract Service Registry Number

RL50 Half-life (also known as Residual Lifetime) of the pesticide on the specified plant matrix

FAO Food and Agriculture Organisation

IUPAC International Union of Pure and Applied Chemistry

PPDB Pesticide Properties Database

US EPA United States Environmental Protection Agency

\section{References}

1. Whitmyre, G.K.; Ross, J.H.; Lunchick, C.; Volger, B.; Singer, S. Biphasic dissipation kinetics for dislodgeable foliar residues in estimating post-application occupational exposures to endosulfan. Arch. Environ. Contam. Toxicol. 2004, 46, 17-23. [CrossRef] [PubMed]

2. Horst, G.L.; Shea, P.J.; Christians, N.; Miller, D.R.; Stuefer-Powell, C.; Starrett, S.K. Pesticide dissipation under golf course fairway conditions. Crop Sci. 1996, 36, 362-370. [CrossRef]

3. Gannon, T.W.; Jeffries, M.D. Dislodgeable 2, 4-D from Athletic Field Turfgrass. Eur. J. Hortic. Sci. 2014, 116-122. Available online: http:/ / www.jstor.org/stable/24126908 (accessed on 3 August 2017).

4. Fantke, P.; Juraske, R.; Jolliet, O. Considering human exposure to pesticides in food products: Importance of dissipation dynamics. In Proceedings of the 9th International Conference on Life Cycle Assessment in the Agri-Food Sector (LCA Food 2014), San Francisco, CA, USA, 8-10 October 2014; American Center for Life Cycle Assessment: Tacoma, WA, USA, 2014; pp. 390-394.

5. Pan, R.; Chen, H.P.; Zhang, M.L.; Wang, Q.H.; Jiang, Y.; Liu, X. Dissipation pattern, processing factors, and safety evaluation for dimethoate and its metabolite (Omethoate) in tea (Camellia Sinensis). PLoS ONE 2015, 10, e0138309. [CrossRef] [PubMed]

6. Chukwudebe, A.C.; Cox, D.L.; Palmer, S.J.; Morneweck, L.A.; Payne, L.D.; Dunbar, D.M.; Wislocki, P.G. Toxicity of emamectin benzoate foliar dislodgeable residues to two beneficial insects. J. Agric. Food Chem. 1997, 45, 3689-3693. Available online: http://pubs.acs.org/doi/abs/10.1021/jf970375c (accessed on 13 April 2017). [CrossRef]

7. Dong, S.; Qiao, K.; Wang, H.; Zhu, Y.; Xia, X.; Wang, K. Dissipation rate of thiacloprid and its control effect against Bemisia tabaci in greenhouse tomato after soil application. Pest Manag. Sci. 2014, 70, 1267-1273. [CrossRef] [PubMed]

8. Zhang, P.; He, M.; Zhao, Y.; Ren, Y.; Wei, Y.; Mu, W.; Liu, F. Dissipation dynamics of clothianidin and its control efficacy against Bradysia odoriphaga Yang and Zhang in Chinese chive ecosystems. Pest Manag. Sci. 2016, 72, 1396-1404. [CrossRef] [PubMed]

9. Fantke, P.; Wieland, P.; Wannaz, C.; Friedrich, R.; Jolliet, O. Dynamics of pesticide uptake into plants: From system functioning to parsimonious modeling. Environ. Model. Softw. 2013, 40, 316-324. Available online: http:/ / doi.org/10.1016/j.envsoft.2012.09.016 (accessed on 13 April 2017). [CrossRef]

10. Shirmohammadi, A.; Knisel, W.G. Evaluation of the GLEAMS model for pesticide leaching in Sweden. J. Environ. Sci. Health A 1994, 29, 1167-1182. Available online: http:/ / dx.doi.org/10.1080/10934529409376101 (accessed on 3 August 2017). [CrossRef]

11. Brouwer, D.H.; De Haan, M.; Leenheers, L.H.; De Vreede, S.A.F.; Van Hemmen, J.J. Half-lives of pesticides on greenhouse crops. Bull. Environ. Contam. Toxicol. 1997, 58, 976-984. Available online: https:/ / www.ncbi. nlm.nih.gov/pubmed/9136663 (accessed on 3 August 2017). [CrossRef] [PubMed] 
12. Juraske, R.; Antón, A.; Castells, F. Estimating half-lives of pesticides in/on vegetation for use in multimedia fate and exposure models. Chemosphere 2008, 70, 1748-1755. Available online: http:/ /doi.org/10.1016/j. chemosphere.2007.08.047 (accessed on 14 July 2017).

13. Fantke, P.; Gillespie, B.W.; Juraske, R.; Jolliet, O. Estimating half-lives for pesticide dissipation from plants. Environ. Sci. Tech. 2014, 48, 8588-8602. [CrossRef] [PubMed]

14. Spynu, E.I. Predicting pesticide residues to reduce crop contamination. Rev. Environ. Contam. Toxcol. 1989, 109, 90-107.

15. Fantke, P.; Juraske, R. Variability of pesticide dissipation half-lives in plants. Environ. Sci. Technol. 2013, 47, 3548-3562. Available online: http:/ / pubs.acs.org/doi/abs/10.1021/es303525x (accessed on 3 August 2017). [CrossRef] [PubMed]

16. Farha, W.; El-Aty, A.A.; Rahman, M.M.; Shin, H.C.; Shim, J.H. An overview on common aspects influencing the dissipation pattern of pesticides: A review. Environ. Monit. Assess. 2016, 188, 693. [CrossRef] [PubMed]

17. Turner, J.A. The Pesticide Manual 17th Edition; British Crop Protection Council: Hampshire, UK, 2015.

18. Lewis, K.A.; Tzilivakis, J.; Warner, D.; Green, A. An international database for pesticide risk assessments and management. Hum. Ecol. Risk Assess. 2016, 22, 1050-1064. Available online: http:/ /dx.doi.org/10.1080/ 10807039.2015.1133242 (accessed on 3 August 2017). [CrossRef]

19. NPIC - National Pesticide Information Center, Oregon State University and the U.S. Environmental Protection Agency. 2017. Available online: http:/ / npic.orst.edu/ (accessed on 3 August 2017).

20. Kegley, S.E.; Hill, B.R.; Orme, S.; Choi, A.H. PAN Pesticide Database. Pesticide Action Network, North America. 2000. Available online: http:/ / www.pesticideinfo.org/ (accessed on 3 August 2017).

21. Severinsen, M.; Jager, T. Modelling the influence of terrestrial vegetation on the environmental fate of xenobiotics. Chemosphere 1998, 37, 41-62. Available online: https:/ / doi.org/10.1016/S0045-6535(98)80002-7 (accessed on 3 August 2017). [CrossRef]

22. Thomas, P.J.; Mineau, P.; Juraske, R. Determining pesticide foliar half-lives from soil half-life value: Not so "cut-and-dry". Chemosphere 2011, 84, 1531-1533. Available online: https:/ / www.ncbi.nlm.nih.gov/pubmed/ 21664644 (accessed on 5 July 2017).

23. Willis, G.H.; McDowell, L.L. Pesticide persistence on foliage. In Reviews of Environmental Contamination and Toxicology; Springer: New York, NY, USA, 1987; pp. 23-73.

24. Katagi, T. Photodegradation of Pesticides on Plant and Soil Surfaces. Rev. Environ. Contam. Toxicol. 2004, 182, 1-195. Available online: https:/ / www.ncbi.nlm.nih.gov/pubmed/15217019 (accessed on 24 July 2017). [PubMed]

25. Hoskins, W.M. Mathematical treatment of loss of pesticide residues. Plant Prot. Bull. (FAO) 1961, 9, 163-168. 